\title{
Group versus Individual Reward in the Asch Experiment without Confederates
}

\author{
Yuki Fujita' ${ }^{1}$ Kazuo Mori $^{2}$ \\ ${ }^{1}$ Teikyo University, Tokyo, Japan \\ ${ }^{2}$ Matsumoto University, Matsumoto, Japan \\ Email: kazuo.mori@t.matsu.ac.jp
}

How to cite this paper: Fujita, Y. and Mori, K. (2017) Group versus Individual Reward in the Asch Experiment without Confederates. Open Journal of Social Sciences, 5, 396-402.

https://doi.org/10.4236/jss.2017.55027

Received: April 19, 2017

Accepted: May 24, 2017

Published: May 27, 2017

Copyright $\odot 2017$ by authors and Scientific Research Publishing Inc. This work is licensed under the Creative Commons Attribution International License (CC BY 4.0).

http://creativecommons.org/licenses/by/4.0/

\begin{abstract}
This study examined whether rewarding participants' principles would affect conformity of the minority responders in the Asch experiment. Twenty groups of four university male students $(\mathrm{N}=80 ; 19$ - 24 years old; mean age, 20.7 and SD, 1.32) participated in the Asch conformity experiments without using confederates, as developed by Mori and Arai (2010). Participants were randomly assigned: one of each foursome to the minority condition and the remaining three to the majority condition. In the half of the groups, the participants were told they would be rewarded individually depending on their each performance (Individual Reward condition). The other half were told to be rewarded group-wise (Group Reward condition). The results showed that the minority responders of the Group Reward groups conformed to the majority, while no minority responders conformed to the majority in the Individual Reward groups.
\end{abstract}

\section{Keywords}

Asch Experiments, Conformity, Rewarding Procedure

\section{Introduction}

The Asch conformity experiments [1] [2] demonstrated that people would conform to the majority opinions when under social pressure. However, the majority responders in the Asch experiments were confederates that acted as the experimenters instructed. Therefore, the participants of the Asch study were in the somewhat strange situation where they were alone among strangers performing the same absurdly simple tasks. Thus, it was doubtful that the participants were so easily fooled by the experimenters and the confederates to believe their perceptual ability was actually being tested.

Although the Asch studies have been replicated with a variety of independent 
variables (culture, sex, response conditions, etc.) for more than a half of century (see [3], for review), many researchers have criticized these studies from various viewpoints. For example, the Asch experiments did not unfold as intended because of a variety of reasons including the unnaturalness of the situation [4]. As for other examples of the criticisms, Stricker, Messick, and Jackson [5] reported that many participants expressed suspicions about the purpose of the Asch experimental procedure. Hodges and Geyer [6] found that even in the original Asch experiment, some participants showed signs of dissent by responding differently on the crucial and control trials in which confederates answered incorrectly and correctly, respectively.

Mori and Arai [7] devised a new experimental procedure utilizing a presentation trick [8] with polarizing filters so that two different stimuli were presented on the same screen without viewers' being aware of the duality. Using this procedure, Mori and Arai presented lines with different lengths to one minority viewer and a group of three others. Since the four viewers were not aware of the presentation trick, the situation was virtually the same as the one created in the original Asch experiment without confederates; the three viewers who observed the different length of the standard line would serve as the source of social pressure. This new procedure reconfirmed that the participants in the isolated condition frequently deferred to the majority.

The original Asch procedure had another weak point because it was incapable to examine the influence of interpersonal variables on conformity. The relationship between the genuine participants and the confederates was obliged to be an unfamiliar one. The Mori and Arai procedure could manipulate the social relationships of the minority and majority in the experimental research on conformity. An individual may conform more frequently to the groups to which she/he belongs than to an unknown group. Even within a familiar group, a member may have stronger social effects from other members when the group has a sense of unity than otherwise. However, it would not be possible to examine this hypothesis with the original Asch procedure.

The present study aimed to examine the effect of a sense of unity among a peer group on conformity by utilizing the Mori and Arai procedure. We manipulated the possible strength of a sense of unity by proposing two different rewarding principles to the participant groups. For the group rewarding condition, participants would receive the reward based on the total number of correct responses of the group divided equally among them. As for the individual rewarding condition, participants would receive individually based on each one's performance. It was hypothesized that the conforming responses would occur more frequently in the group rewarding condition than in the individual condition.

\section{Method}

\subsection{Participants}

Eighty university male students (19 to 24 years old, 20.7 and 1.32, for mean and $\mathrm{SD}$, respectively) majoring in various areas of engineering and technology parti- 
cipated. They were peers and came to the laboratory in a group of four by having read the psychology experiment advertisement on the campus bulletin board. All of them had normal or corrected-to-normal visions.

\subsection{Experimental Design}

The experimental design was a 2 (role: minority vs. majority) $\times 2$ (reward principle: group, individual) between-subjects factorial design. Each group was assigned randomly to the Group and Individual reward conditions. In each group, the response order was randomly decided before entering the laboratory, and the third responder was assigned to the minority condition presented the lines differently from those of the other three. The dependent variable was the frequency of errors in the 12 critical tasks.

Reward conditions. In Group Reward condition, each participant was paid an equal amount of money having divided the total reward calculated based on the total number of correct responses made by the group; $¥ 50$ (about US\$0.50) for each correct response. As for Individual Reward condition, each participant was paid an amount calculated based on the total number of correct responses; $¥ 50$ for each correct response. There were 18 trials, so the maximum amount of reward would be $¥ 900$. However, all the participants were paid $¥ 1000$ (about US $\$ 10.00)$ after the tasks irrespective of their actual performance.

Stimuli. The same set of stimuli used by Mori and Arai [7] were used. The stimuli were created to be equivalent to the nine stimulus sets that Asch [2] had used. Six of the nine sets were made for the critical tasks in which the minority viewer would see the standard lines differently from the majority viewers. The remaining three sets were made for the neutral trials in which the same stimuli were presented to the minority and majority students, corresponding to the neutral condition in the original Asch study in which confederates answered correctly.

\subsection{Apparatus}

The stimuli were presented on PowerPoint slides with a personal computer and projected by an LCD projector onto a rear screen. The rear screen was made of a pane of ground glass measuring $80 \mathrm{~cm} \times 80 \mathrm{~cm} \times 0.5 \mathrm{~cm}$. The rear screen was set about $1 \mathrm{~m}$ away from the projector (see Figure 1).

\subsection{Procedure}

The procedure followed that of Mori and Arai [7] with one minor modification. Instead of letting participants respond during the presentation of stimuli, participants were instructed to respond after the presentation.

Participants came to the laboratory in a group of four. Before entering the laboratory, participants were asked to decide their answering order using the "Rock-paper-scissors" procedure. Then, they entered the laboratory in the determined order and took the corresponding chair numbered \#1 to \#4. They then picked up a pair of polarizing sunglasses placed on the chair and put them on. 
Participants were told the sunglasses were to protect their eyes from glare (cover story).

Then, the following instructions were given by the experimenter and also presented on the screen:

This is a task involving the discrimination of lengths of lines. In front of you is a screen. On the left of the screen, there will be one line, and on the right there will be three lines differing in length; they are numbered 1, 2, and 3 , in order. One of the three lines at the right is equal to the standard line at the left. You will decide which is the equal length line in each task. You will state your judgment in terms of the number of the line. There will be 18 comparisons in all. As the number of comparisons is few and the group small, I will call upon each of you in turn to announce your judgments, which I will record here on a prepared form. Since your seat order was determined, you will give your answer in the seating order, from \# 1 to \# 4 .

Following this, the experimenter stated that participants were to answer as accurately as possible, to make the judgment by themselves, not to talk or react to the others, and to stay quiet except when it was the participant's turn to answer.

Then, the line judgment trials started in the predetermined order. As in the Asch [2] study, the trials comprised a series of the nine tasks repeated twice. Each trial took approximately 15 seconds.

After the tasks, a questionnaire was administered, asking whether participants noticed any abnormalities in the presented slides. Then, the experimenter paid the participants $¥ 1000$ each and debriefed them about the purpose of the study, including the presentation trick used. The whole session took about 30 minutes.

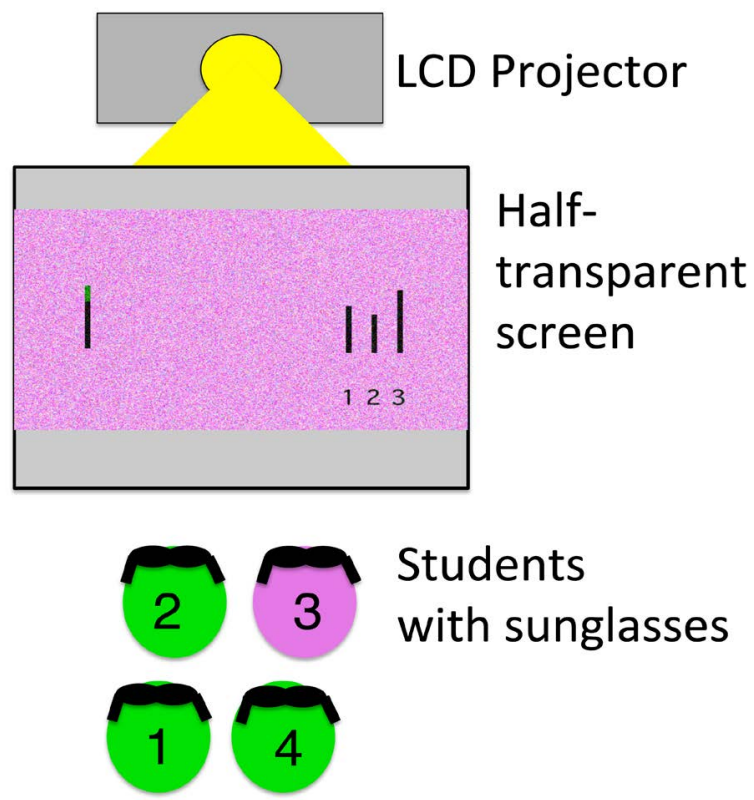

Figure 1. Diagram of the experimental setting. The top green part of the standard line appears in black with the green-broking sunglasses while disappears with the magentabroking sunglasses. 


\section{Results}

\subsection{Errors Made by the Minority and Majority Participants}

Unlike the original Asch experiments, the genuine participants in the majority condition of the present study would make errors. Therefore, we first analyzed the number of errors under the majority and minority conditions (Table 1). According to Table 1, there were more errors made by the third responders, i.e., the minority participants, than by the other three responders $\left(X_{(3)}^{2}=33.94, p<\right.$ 0.01 , Cramer's $V=0.188$ ). Following to Mori and Arai [7], the excessing number of errors in the third responders was regarded as the index of conformity. The results showed that the experimental procedure elicited conforming behavior of the minority participants, as expected.

\subsection{Effect of Rewarding Principles}

Table 2 shows the response patterns of the minority participants in the two experimental conditions. There were three choices in each task for the minority participants; the correct choice, the error choice conforming to the majority, and the other error choice. It should be noted that there were inappropriate cases where the intended social pressure was not created because of occasional errors in the responses of the first and second responders. We discarded those cases from the analysis. The errors made by the fourth responders were disregarded because they were made only after the responses of the minority.

The results clearly showed that there were more conforming responses in the Group condition than the Individual condition. There was only one case of conforming response in the Individual condition $\left(X_{(1)}^{2}=20.66, p<0.01, \varphi=0.372\right)$. These results supported the experimental hypothesis of the present study.

As Table 2 shows, there were considerable discarded cases because of the errors made by the majority participants. It was suspected that the majority par-

Table 1. Total numbers of errors sorted for the four responding orders (the third responders were the minority participants).

\begin{tabular}{cccccc}
\hline Order & 1st & 2nd & 3rd & 4 th & Total \\
\hline Errors & 34 & 28 & 62 & 19 & 143 \\
Corrects & 206 & 212 & 178 & 221 & 817 \\
Total & 240 & 240 & 240 & 240 & 960 \\
\hline
\end{tabular}

Table 2. The Number of Responses on the All the Critical Tasks.

\begin{tabular}{cccccc}
\hline \multirow{2}{*}{ Conditions } & \multicolumn{5}{c}{ Responses } \\
\cline { 2 - 6 } & Correct & Conformity & Other & Total & Discarded $^{\text {a) }}$ \\
\hline Group & 60 & 33 & 9 & 102 & 18 \\
Individual & 55 & 1 & 12 & 68 & 52 \\
Total & 115 & 34 & 21 & 170 & 70
\end{tabular}

a. The data discarded because of the errors in the first and second responders. 
ticipants made errors intentionally to attenuate the embarrassing situations because otherwise there would be only one of their friends who made errors repeatedly. Therefore, the data were re-analyzed using only the first critical trial to avoid the bias from the intentional errors. Please note that until the first critical task, the participants had not experienced any differences among their responses because they had been given only neutral tasks. Owing to the small sample size, we could report here only the numbers of conformity responses in the two rewarding conditions. There were two conformities found among ten participant groups in the Group condition whereas none in the Individual condition. (Fisher's exact test; $p=0.24$, ns, $\varphi=0.333$ ).

\section{Discussion}

We successfully reproduced a situation equivalent to the Asch conformity experiment without using confederates. We also manipulated the group dynamics among the responders including the majority participants by introducing the two different rewarding principles. The different reward procedures resulted in different conforming tendencies among the participants. Mori and Arai [7] found that Japanese male students did not conform to the majority. However, the present study showed that Japanese male students did conform to the majority when the reward would be paid group-wise.

The Mori and Arai procedure is the only available methodology to examine the social effects of interpersonal relations on the behavior of the minority person in the Asch paradigm. Mori and Uchida [9] utilized this procedure to investigate the effect of scholastic achievement levels between the minority and majority students of a junior high school. They found that a low-achievement student surrounded by high-achievement peers tended to conform to them. Mori, Ito-Koyama, Arai, and Hanayama [10] applied this procedure to the three age groups and found a developmental sex difference in conformity. There are many more social factors left to be examined with this new procedure.

Although there are promising potentials of the Mori and Arai procedure for re-examining the results having obtained with the original Asch experiments, there are limitations as well. According to the questionnaire conducted after the tasks, some participants noticed the trick. The participants in the present study were university students majoring in engineering and technology. They might have had knowledge of polarization of light. However, they seemed to have figured out the trick principle only in the middle of the session. The number of errors rose sharply after the third critical task especially in the individual group, from one in the third task to eight in the fourth. The single-trial version would be less vulnerable to the risk of detection by the viewers. It would also be recommended to place the minority responders in the fourth order rather than the third as in the previous studies including the present one.

\section{Acknowledgements}

This research was supported by a Grant-in-Aid from the Japan Society for the 
Promotion of Science (JSPS) (KAKENHI 21012002 \& 23011004) to the second author while working at the Tokyo University of Agriculture and Technology (TUAT). This research was approved by the ethical committee of the TUAT. We thank all the students for participating in the experiment. Part of this study was presented in Japanese at the $16^{\text {th }}$ Conference on Experimental Social Sciences held at Aoyama University, Tokyo, in December 2012.

\section{References}

[1] Asch, S.E. (1951) Effects of Group Pressure on the Modification and Distortion of Judgments. In Guetzknow, H., Ed., Groups, Leadership and Men, Pittsburgh, PA, Carnegie Press, 177-190.

[2] Asch, S.E. (1956) Studies of Independence and Conformity: I. A Minority of One against a Unanimous Majority. Psychological Monograph: General and Applied, 70, Whole No. 416.

[3] Bond, R. and Smith, P.B. (1996) Culture and Conformity: A Meta-Analysis of Studies Using Asch's (1952b, 1956) Line Judgment Task. Psychological Bulletin, 119, 111-137. https://doi.org/10.1037/0033-2909.119.1.111

[4] Standing, L.G. and Lalancette, M.-F. (1990) Asch Fails Again. Social Behavior and Personality: An International Journal, 18, 7-12.

https://doi.org/10.2224/sbp.1990.18.1.7

[5] Stricker, L.J., Messick, S. and Jackson, D.N. (1967) Suspicion of Deception: Implications for Conformity Research. Journal of Personality and Social Psychology, 5, 379389. https://doi.org/10.1037/h0021209

[6] Hodges, B.H. and Geyer, A. (2006) A Nonconformist Account of the Asch Experiments: Values, Pragmatics, and Moral Dilemmas. Personality and Social Psychology Review, 10, 2-19. https://doi.org/10.1207/s15327957pspr1001_1

[7] Mori, K. and Arai, M. (2010) No Need to Fake It: Reproduction of the Asch Experiment without Confederates. International Journal of Psychology, 45, 390-397. https://doi.org/10.1080/00207591003774485

[8] Mori, K. (2007) Projecting Two Words with One Machine: A Method for Presenting Two Different Visual Stimuli Using Just One Projector without Viewer' Noticing the Duality. Behavior Research Methods, 39, 811-815. https://doi.org/10.3758/BF03192973

[9] Mori, K. and Uchida, A. (2013) Conformity and Scholastic Levels: An fMORI-Asch Experimental Study of Japanese Junior High School Students. Paper Presented at the 10th Biennial Meeting of the Society for Applied Research in Memory and Cognition (SARMAC X), Rotterdam, The Netherlands.

[10] Mori, K., Ito-Koyama, A., Arai, M. and Hanayama, A. (2014) Boys, Be Independent! Conformity Development of Japanese Children in the Asch Experiment without Using Confederates. Psychology, 5, 617-623.

https://doi.org/10.4236/psych.2014.57073 
Submit or recommend next manuscript to SCIRP and we will provide best service for you:

Accepting pre-submission inquiries through Email, Facebook, LinkedIn, Twitter, etc. A wide selection of journals (inclusive of 9 subjects, more than 200 journals)

Providing 24-hour high-quality service

User-friendly online submission system

Fair and swift peer-review system

Efficient typesetting and proofreading procedure

Display of the result of downloads and visits, as well as the number of cited articles Maximum dissemination of your research work

Submit your manuscript at: http://papersubmission.scirp.org/

Or contact jss@scirp.org 